\title{
KAM theory for particles in periodic potentials
}

\author{
MARK LEVI $\dagger$ \\ Department of Mathematıcs, Boston University, Boston, Mass 02215, USA
}

(Recelved 19 Aprll 1988)

\begin{abstract}
It is shown that the system of the form $x+V^{\prime}(x)=p(t)$ with periodic $V$ and $p$ and with $\langle p\rangle=0$ is near-integrable for large energies In particular, most (in the sense of Lebesgue measure) fast solutions are quasıperiodic, provided $V \in C^{(5)}$ and $p \in L^{1}$, furthermore, for any solution $x(t)$ there exists a velocity bound $c$ for all tıme $|x(t)|<c$ for all $t \in \mathbf{R}$ For any real number $r$ there exists a solution with that average velocity, and when $r$ is rational, this solution can be chosen to be periodic
\end{abstract}

\section{Introduction}

The result of this note states that any system consisting of a particle in a periodic potential subject to periodic external forcing possesses KAM tori, physically corresponding to quasiperiodic translational motion The only assumptions are the $C^{(5)}$ smoothness of the potential and the zero average of the forcing In particular, forcing need not be small This note was stımulated by J Franks' extension of the PoincaréBirkhoff theorem to the case when the boundaries of the annulus are noninvariant This extension was proven and applied (the latter in collaboration with Moeckel and Robinson) in [5] to the conservative pendulum with periodic forcing

$$
x+\sin x=p(t), \quad \int_{0}^{1} p(t) d t=0,
$$

where $p(t+1)=p(t)$, to show that periodic solutions of any rational rotation number exist

The standard version [2] of the Poincaré-Birkhoff theorem may not seem appl1cable in this case since no annulus with invariant boundaries is available a priori We will show that such boundaries actually do exist, using Moser's invariant curve theorem [8], [13], [16]

We will look at the systems of the form

$$
x+V^{\prime}(x)=p(t), \quad V(x+1)=V(x), \quad p(t+1)=p(t)
$$

describing the motion of a particle in a periodic potential with external periodic forcing $p(t)$ This could be a simple model of an electron in an atomic lattice subject 
to a periodically varying potential, or of charge-density waves [6] We will show that the associated Poincaré map $F(x, x)_{t=0} \mapsto(x, x)_{t=1}$ of the phase cylinder $(x \bmod 1, x)$ onto itself possesses noncontractible invariant circles The results are stated fully in $\S 2$ We mention a related result by Zehnder and Dieckerhoff $[3,4]$ on the existence of KAM circles for the particle in a superquadratic potential

$$
\begin{aligned}
& x+V_{x}(x, t)=0, \\
& V(x, t)=x^{2 n}+a_{1} x^{2 n-1}+\quad+a_{2 n}, \quad n>1
\end{aligned}
$$

with lower coefficients $a_{i}(t)$ periodic in $t$ The superquadratic nature of the potential provided the twist needed to apply KAM In the case at hand, the twist is provided by a different effect, essentially, by the shear in the $(x, x)$-plane whose physical manifestation lies in the obvious fact that the faster particles travel further The invariant circles on the phase torus turn out to be approximately straight for large energies, this elımınates the need of a prelımınary change into action-angle variables, makıng the proofs simpler than in the superquadratic case (3)

We mention also the earlier results by Jacobowitz and Struble [9] and by Hartman [7], where the superquadratic nature of the potential was used to apply the PoincaréBirkhoff theorem to prove the existence of periodic solutions in a class of systems of the form (3)

\section{Results}

THEOREM 1 Assume that $V(x) \in C^{(5)}$ and that $p(t)$ is continuous (actually, summable is enough) with $\int_{0}^{1} p(t) d t=0$

For any $0<\omega<1$ sattsfying for some $c_{0}>0$ and $\mu>0$ the set of inequalities

$$
\left|\omega-\frac{m}{n}\right|>\frac{c_{0}}{n^{2+\mu}} \quad \forall m, n \in \mathbf{Z}, n \neq 0,
$$

there exists an integer $k_{0}=k\left(c_{0}, \mu\right)>0$ such that Poincaré map $F(x, x)_{t=0} \mapsto(x, x)_{t=1}$ of eq (2) possesses a countable set of invariant curves $y=f_{\omega+k}(x) \equiv f_{\omega+k}(x+1)$, for all integers $|k| \geq k_{0}$ with translation numbers $\omega+k$ The corresponding invartant circles in the phase cylinder $(x \bmod 1, x)$ have rotation numbers $\omega$ For large $|k|$ we have

$$
f_{\omega+k+1}(x)-f_{\omega+k}(x)=1+O\left(k^{-1}\right) \text { and } f_{\omega+k}^{\prime}=O\left(k^{-1}\right)
$$

Relative measure of invariant circles in an annulus $N \leq x \leq N+1$ tends to one as $N \rightarrow \infty$

Applying a recent result of Herman [8] on the existence of invariant curves for $C^{(3)}$-small perturbations of the twist maps, one can lower the smoothness assumption to $V \in C^{(4)}$, at the expense of replacing the Diophantine condition on $\omega$ by the requirement that $\omega$ be of constant type One also loses the statement on the relative measure of invariant circles

Each such invariant circle sweeps out an invariant torus in the extended phase space $\{(x \bmod 1, x, t \bmod 1)\}=S^{1} \times \mathbf{R} \times S^{1} \quad$ Each orbit on such a torus is quasiperiodic with basic frequencies 1 and $\omega+k$. 
Physically, these orbits correspond to quasiperiodic rotations with average angular velocity $\omega+k$, or to quasiperiodic translations (in the potential well interpretation) with the average speed $\omega+k$ The basic frequencies of the quasiperiodic solutions are 1 and $\omega+k$.

The existence of invariant circles implies at once the following

COROLlary 1 Any solution of (2) is bounded in the phase cylinder $\{(x \bmod 1, x)\}=$ $S^{1} \times \mathbf{R}$, in other words, any solution of (2) has bounded angular veloctty

Since Poincaré map $F$ defined above is a composition of monotone twist maps, we can apply the Aubry-Mather theorem [1, 12, 13], obtainıng

COROLLARY 2 For any real number $\omega$ there exists a Birkhoff orbit with that rotation number Physically, there exists a motion with any average angular velocity Furthermore, as a consequence of the Poincaré-Bırkhoff's theorem, for any rational $\omega=p / q$ there exists a periodic solution satisfying $x(t+q)=x(t)+q$

A note added in proof After this paper had been submitted I learned that Jurgen Moser had proved a similar statement for a more general equation $x+W_{x}(x, t)=0$ with $W_{x}$ periodic in both arguments, satisfying the exactness condition $\int_{0}^{1} \int_{0}^{1} W_{x}(x, t) d x d t=0$ and smooth enough [17] Moser's proof is based on a varnational approach, the proof given in the present note is different-it is based on the application of Moser's invariant curve theorem [13] The proof given here carnes over almost verbatım to the more general case when the dependence on $x$ and $t$ is not separate The argument requires, however, the differentiability in $t$ as well, as It does in [17], it suffices to assume $V \in C^{(5)}\left(\mathbf{R}^{2}\right)$ In the special case (2) no smoothness in $t$ is assumed

\section{Proof}

We will show that $F$ is an exact map of the cylinder which is $C^{(4)}$-close to the linear shear for large $|y|=|x|$

\section{Exactness of the Poincaré map}

The exactness of the cylinder map $F$ is due, as it turns out, to the zero-average property of $p(t)$ - this was observed previously by Moeckel, Robinson [5] and others For completeness, we provide a proof

Let $C_{0}$ be an arbitrary noncontractıble circle going once around the cylinder, and let $C_{0}$ be its image under the flow of eq (2)

$$
z=f(z, t), \quad z=\left(\begin{array}{l}
x \\
y
\end{array}\right), \quad f=\left(\begin{array}{c}
y \\
-V^{\prime}(x)+p(t)
\end{array}\right)
$$

Thus $C_{1}=F C_{0}$, we have to show that

$$
\int_{C_{0}} y d x=\int_{C_{1}} y d x
$$

Let $A$, be the annulus bounded by the horizontal circle $H=\{y=0\}$ of the $x$-axis (oriented in the positive direction) and by $C_{t}$ (oriented in the opposite direction to the $x$-axis), figure 1 


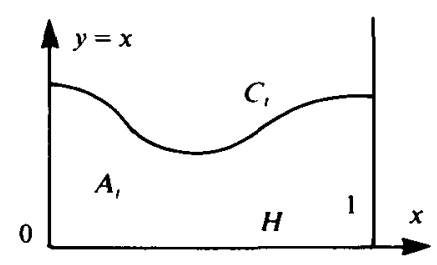

Figure 1 Proof of exactness

Differentiating the area $\iint_{A_{t}} d x d y$ and observing that only one component $C_{t}$ of the boundary is moving with the flow (4), we obtain

$$
\frac{d}{d t} \iint_{A_{t}} d x d y=-\int_{C_{t}} f n d s,
$$

where $n$ is the outward unit normal vector

Using the divergence theorem, we rewrite the flux on the right-hand side as

$$
\begin{aligned}
-\int_{C_{t}} f n d s & =\int_{H} f n d s-\iint_{A_{t}} \operatorname{div} f d x d y \stackrel{(\mathrm{A})}{=} \int_{H} f n d s \\
& =\int_{0}^{1} V^{\prime}(x) d x+\int_{0}^{1} p(t) d x=\int_{0}^{1} p(t) d t \stackrel{(\mathrm{B})}{=} 0,
\end{aligned}
$$

where the Hamiltonian (1 e, divergence-free) character of $f$ was used in (A) and zero-average property of $p$ was used in (B) We conclude that

$$
\iint_{A_{0}} d x d y=\iint_{A_{1}} d x d y
$$

which by Stokes' theorem is equivalent to the desired exactness property (5)

\section{Near-integrability}

To prove Theorem 1 it remains to show that the map $F$ is $C^{(4)}$-close to the twist for large $y$, we will show in fact, that

$$
F\left(\begin{array}{l}
x \\
y
\end{array}\right)=\left(\begin{array}{ll}
1 & 1 \\
0 & 1
\end{array}\right)\left(\begin{array}{l}
x \\
y
\end{array}\right)+\left(\begin{array}{l}
\bar{P} \\
0
\end{array}\right)+r(x, y),
$$

where $\bar{P}=\int_{0}^{1} \int_{0}^{1} p(\tau) d \tau d t$ and $r=\operatorname{col}\left(r_{1}, r_{2}\right)$ is $C^{(4)}$-small there exists a constant $C$ depending on $V()$ and $p()$ only, such that

$$
\left\|r_{1}\right\|_{C^{4}}, \quad\left\|r_{2}\right\|_{C^{4}}<C|y|^{-1}
$$

The idea of the proof is to show that for high angular velocities the effect of periodic potential averages out to zero

To prove (6) and (6a) we let $z(t) \equiv z\left(t, s_{1}, s_{2}\right)$ be the solution of (4) with $z\left(0, s_{1}, s_{2}\right)=\operatorname{col}\left(s_{1}, s_{2}\right)$ To estimate $r(x, y)$ we introduce the derivative vectors

$$
z_{\imath}=\frac{\partial z}{\partial s_{i}}, \quad z_{\imath}=\frac{\partial^{2} z}{\partial s_{\imath} \partial s_{j}}, \quad z_{i j h}=\frac{\partial^{3} z}{\partial s_{\imath} \partial s_{\jmath} \partial s_{k}}, \quad z_{\imath h l}=\frac{\partial^{4} z}{\partial s_{\imath} \partial s_{\jmath} \partial s_{k} \partial s_{l}},
$$


where $l, J, k, l=1$ or 2 and show that for $t=1$

$$
z_{1}(1)=\left(\begin{array}{l}
1 \\
0
\end{array}\right)+O\left(|y|^{-1}\right), \quad z_{2}(1)=\left(\begin{array}{l}
1 \\
1
\end{array}\right)+O\left(|y|^{-1}\right),
$$

while

$$
\left|z_{\imath}(1)\right|,\left|z_{\imath j k}(1)\right|,\left|z_{\imath \jmath k l}(1)\right|=O\left(|y|^{-1}\right) \quad \text { for } y \text { large }
$$

These vectors satisfy the linear, Hessian cubic and quartic versions of eq (4)

$$
\begin{aligned}
& z_{1}=f_{z}(z, t) z, \quad z_{1}(0)=\left(\begin{array}{l}
1 \\
0
\end{array}\right), \quad z_{2}=\left(\begin{array}{l}
0 \\
1
\end{array}\right), \\
& z_{\imath \jmath}=f_{z} z_{\imath \jmath}+f_{z z}(z)\left[z_{\imath}, z_{\jmath}\right], \quad z_{\imath \jmath}(0)=0, \\
& z_{i j k}=f_{z} z_{i j k}+f_{z z}\left[z_{k}, z_{i j}\right]+f_{z z}\left[z_{i}, z_{j k}\right]+f_{z z}\left[z_{i k}, z_{j}\right]+f_{z z}\left[z_{k}, z_{i}, z_{j}\right], \quad z_{i j k}(0)=0,
\end{aligned}
$$

and simılarly for $z_{i j k l}$ The derivatives $f_{z z}, f_{z z z}$ and $f_{z z z z}$ are tensors (multilınear functions of their arguments) with coefficients being second, third and fourth mixed partials in $x$ and $y$ of the components of $f(z)$ For $f(z, t)$ as in (4), these depend only on $V^{(\prime)}(x)$ with $t \leq 5$ and thus satisfy, for some $C>0$ and for all $x \in \mathbf{R}$, the bounds

$$
\begin{aligned}
\left|f_{z z}[u, v]\right| & \leq C|u \| v| \\
\left|f_{z z z}[u, v, w]\right| & \leq C|u||v||w| \\
\left|f_{z z z z}[u, v, w, \zeta]\right| & \leq C|u||v||w||\zeta|
\end{aligned}
$$

To obtain the desired $C^{(4)}$-estimate $(6 a)$ on the remainder we will use the following estımate on $z\left(t, x_{0}, y_{0}\right) \equiv z\left(t, z_{0}\right)$ valid for large $\left|y_{0}\right|$, given in Lemma 1 and in its two corollanes

\section{Auxiliary lemmas}

LEMMA 1 Let $B=\max _{x}\left|V^{\prime}(x)\right|+\max _{t}|p(t)|$ For any $x_{0}, y_{0}, t$ with $y_{0} \geq 8 B$ and $0 \leq t \leq 1$, the solution $z\left(t, z_{0}\right)$ of (4) lies in the triangle shown in figure $2, t e$ satisfies

$$
\begin{gathered}
y_{0}+\frac{2 B}{y_{0}}\left(x\left(t, z_{0}\right)-x_{0}\right) \geq y\left(t, z_{0}\right) \geq y_{0}-\frac{2 B}{y_{0}}\left(x\left(t, z_{0}\right)-x_{0}\right), \\
x_{0} \leq x\left(t, z_{0}\right) \leq x_{0}+2 y_{0}
\end{gathered}
$$

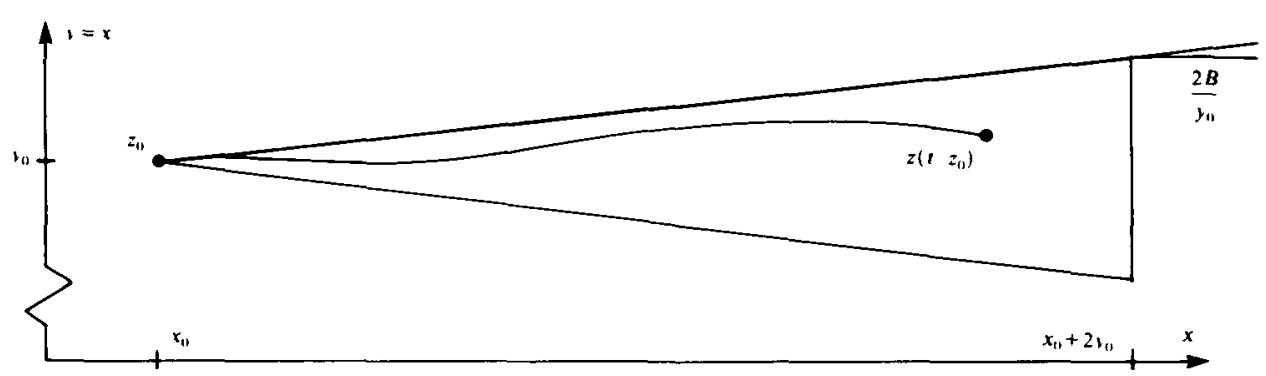

FigURE 2 Estimate on $z\left(t, z_{0}\right)$ as given by Lemma 2 
Proof of Lemma 1 We show that the solution $z\left(t, z_{0}\right)$ stays in the triangle defined by (9) during $0 \leq t \leq 1$ by proving first that $z\left(t, z_{0}\right)$ cannot leave through the sloped sides

$$
y=y_{0} \pm \frac{2 B}{y_{0}}\left(x-x_{0}\right) \stackrel{\text { def }}{=} L_{ \pm}(x), \quad x_{0} \leq x \leq x_{0}+2 y_{0},
$$

since the flow crosses these inwards First we show that the lower boundary is crossed upwards if $y(t)-L_{-}(x(t))=0$ and $x_{0} \leq x(t) \leq x_{0}+2 y$, then

$$
\begin{aligned}
\frac{d}{d t}\left[y-\left(y_{0}-\frac{2 B}{y_{0}}\left(x-x_{0}\right)\right)\right] & =-V^{\prime}+p+\frac{2 B}{y_{0}} y \\
& =-V^{\prime}+p+\frac{2 B}{y_{0}}\left(y_{0}-\frac{2 B}{y_{0}}\left(x-x_{0}\right)\right) \\
& >-B+2 B-\frac{4 B^{2}}{y_{0}^{2}}\left(x-x_{0}\right) \\
& >B-\frac{4 B^{2}}{y_{0}^{2}} 2 y_{0}=B-\frac{8 B^{2}}{y_{0}}>0, \text { since } y_{0}>8 B
\end{aligned}
$$

Second, the top boundary of the triangle defined by (9) is crossed downwards for points $(x, y)$ on the top boundary segment we have

$$
\begin{aligned}
\frac{d}{d t}\left[y-L_{+}(x)\right] & =\frac{d}{d t}\left[y-\left(y_{0}+\frac{2 B}{y_{0}}\left(x-x_{0}\right)\right)\right]=-V^{\prime}+p-\frac{2 B}{y_{0}} y \\
& =-V^{\prime}+p-\frac{2 B}{y_{0}}\left(y_{0}+\frac{2 B}{y_{0}}\left(x-x_{0}\right)\right) \\
& <B-2 B-\frac{4 B^{2}}{y_{0}^{2}}\left(x-x_{0}\right)<-B<0
\end{aligned}
$$

Since $z(t)$ stays below the line, it does not travel too fast to the right - this gives the estimate on $x(t)$

$$
x=y<y_{0}+\frac{2 B}{y_{0}}\left(x-x_{0}\right)
$$

and thus

$$
x(t) \leq x_{0}+\frac{y_{0}^{2}}{2 B}\left(e^{2 B t / t_{0}}-1\right) \quad \text { for } t \geq 0,
$$

which for $0 \leq t \leq 1$ and $y_{0} \geq 8 B$ gives

$$
x(t) \leq x_{0}+2 y_{0} t
$$

COROLLARY 3 There exists a constant $C>0$ independent of $\left(x_{0}, y_{0}\right)$ such that for all $\left|y_{0}\right| \geq 8 B$ and for all $x_{0}$

$$
\left|\int_{0}^{1} V^{(k)}\left(x\left(t, x_{0}, y_{0}\right)\right) d t\right| \leq C\left|y_{0}\right|^{-1}, \quad k=1,2,3,4,5
$$

Proof of Corollary 3 The idea of the proof is simple if $x(t)$ had changed linearly with time, the integral in question would have been zero Since $x(t)$ changes 
near-linearly by Lemma 1 , the integral should be small To make this rigorous, we choose $x$ as the independent variable, obtaining

$$
\begin{aligned}
\left|\int_{0}^{1} V^{(k)}(x(t)) d t\right| & =\left|\int_{x_{0}}^{x_{1}} V^{(k)}(x) \frac{1}{y(x)} d x\right| \\
& =\left|\frac{V^{(k)}(x)}{y(x)}\right|_{x_{0}}^{x_{1}}-\int_{x_{0}}^{x_{1}} \frac{V^{(k-1)}(x) y^{\prime}(x)}{u^{2}(x)} d x \mid \leq \frac{C}{\left|y_{0}\right|},
\end{aligned}
$$

where Lemma 1 was used to estımate $y^{2} \geq C y_{0}^{2}$ from below and the estımates

$$
\left|y^{\prime}(x)\right|=\left|\frac{d y}{d x}\right|=\left|\frac{-V^{\prime}+p}{y}\right| \leq \frac{C}{\left|y_{0}\right|} \quad \text { and } \quad\left|x_{1}-x_{0}\right|<2 y_{0}
$$

were incorporated, both following from Lemma 1

Corollary 4 Let $g[\mathbf{0}, 1] \rightarrow \mathbf{R}$ be $C^{1}$-bounded $|g(t)|,\left|g^{\prime}(t)\right|<C_{1}$, let $V \in C^{(5)}$ and assume that $z\left(t, z_{0}\right)$ satisfies conclusion (9) of Lemma 1 Then $\exists C>0$ such that for all $y_{0} \geq 8 B$ we have

$$
\left|\int_{0}^{1} g(t) V^{(k)}(x(t)) d t\right|<C\left|y_{0}\right|^{-1}, \quad 1 \leq k \leq 5
$$

Proof of Corollary 4 The idea of the proof is similar to the one above The added ingredient (implicit in our analytic argument) is the fact that $g$ changes slowly with respect to $x(t)$ so that the integrand averages out to near-zero over one full $2 \pi$-rotation of $x$

With $h(x) \equiv V^{(k-1)}(x)$, we have, choosing $x$ as the independent variable

$$
\begin{aligned}
\left|\int_{0}^{1} g V^{(k)} d t\right| & =\left|\int_{0}^{1} g h^{\prime}(x(t)) d t\right|=\left|\int_{0}^{1} g(t) \frac{h^{\prime}(x(t))}{y(x(t))} x d t\right| \\
& =\left|\int_{x_{0}}^{x_{1}} h^{\prime}(x) \frac{g(t(x))}{y(x)} d x\right| \\
& =\left|h(x) \frac{g(t(x))}{y(x)}\right|_{x_{0}}^{x_{1}}-\int_{x_{0}}^{x_{1}} h(x) \frac{g^{\prime} d t / d x y(x)-g(t(x)) y^{\prime}(x)}{y^{2}(x)} d x \mid \\
& \leq\left.\left|h(x) \frac{g(t(x))}{y(x)}\right|\right|_{x_{0}} ^{x_{1}}|+| x_{1}-x_{0} \mid \frac{\max \left|g^{\prime}-g y^{\prime}\right|}{\min y^{2}}
\end{aligned}
$$

Since $h, g$ are bounded and $|y| \geq\left|y_{0}\right|-4 B$ (Lemma 1), the first term in the last sum satısfies the desired estimate Similarly, the second term is less than $C\left|y_{0}\right|^{-1}$ since $\left|x_{1}-x_{0}\right| \leq 2\left|y_{0}\right|$, and

$$
\left|y^{\prime}(x)\right|=\left|\frac{d y}{d x} \frac{d t}{d x}\right|=\left|\frac{y}{x}\right|=\left|\frac{-V^{\prime}+p}{y}\right| \leq \frac{B}{|y|}
$$

\section{End of the proof of Theorem 1}

We now begin the end of the proof of the theorem

As the first step, we estimate the position $z\left(1, z_{0}\right)$ after one period Integrating the second component of eq (3), we obtain for $0 \leq t \leq 1$

$$
y(t)=y_{0}+\int_{0}^{\prime}\left(-V^{\prime}+p\right) d t=y_{0}+\int_{0}^{t} p(\tau) d \tau+O\left(\frac{1}{\left|y_{0}\right|}\right),
$$


using Corollary 3 , in particular, for $t=1$ we obtain

$$
y(1)=y_{0}+O\left(\left|y_{0}\right|^{-1}\right)
$$

Furthermore, integrating (12), we obtain

$$
\begin{aligned}
x(1)=x_{0}+\int_{0}^{1} y(t) d t & =x_{0}+\int_{0}^{1}\left[y_{0}+\int_{0}^{t} p(\tau) d \tau+O\left(\left|y_{0}\right|^{-1}\right)\right] d t \\
& =x_{0}+y_{0}+\vec{P}+O\left(\left|y_{0}\right|^{-1}\right)
\end{aligned}
$$

This proves the $C^{0}$-smallness of the remainder $r$ in (6) To get the $C^{1}$-estimate, we evaluate $z_{1}(1)$ introducing matrices $A(t)=f_{z}(z(t))$ and $B(t)=\int_{0}^{t} A(\tau) d \tau$, we obtain from the linearized equation $(7 \mathrm{a})$

$$
\begin{aligned}
z_{l}(1) & =z_{\imath}(0)+\int_{0}^{1} A(t) z_{\imath}(t) d t=z_{\imath}(0)+\int_{0}^{1} B^{\prime}(t) z_{\imath}(t) d t \\
& =z_{l}(0)+B(1) z_{l}(1)-\int_{0}^{1} B(t) A(t) z_{\imath}(t) d t
\end{aligned}
$$

the last step involved integration by parts Using Corollary 3 on the elements of the matrix $B$ we obtain

$$
B(1)=\left(\begin{array}{ll}
0 & 1 \\
0 & 0
\end{array}\right)+O\left(\left|y_{0}\right|^{-1}\right),
$$

while Corollary 4 gives

$$
\int_{0}^{1} B(t) A(t) z_{1}(t) d t=O\left(\left|y_{0}\right|^{-1}\right),
$$

resulting in

$$
z_{i}(1)=\left(\begin{array}{ll}
1 & 1 \\
0 & 1
\end{array}\right) z_{i}(0)+O\left(\left|y_{0}\right|^{-1}\right),
$$

proving the $C^{1}$-estimate on $r$

The last argument shows also that the fundamental solution matrix $\Phi$ for the linearized equation $(7 \mathrm{a})$ satisfies

$$
\Phi(t, \tau)=\left(\begin{array}{cc}
1 & t-\tau \\
0 & 1
\end{array}\right)+O\left(\left|y_{0}\right|^{-1}\right), \quad 0 \leq \tau \leq t \leq 1
$$

This will be used in the remaining estimates

Equation (7b) is inhomogeneous in $z_{i l}$, but the inhomogeneity has been estımated in the previous steps To show that $\left.z_{y}(1)=\left.O\left(\mid y_{0}\right)\right|^{-1}\right)$, we use the variation of constant formula to obtain

$$
\begin{aligned}
z_{l \jmath}(1) & =z_{\imath \jmath}(0)+\Phi(1,0) z_{\imath \jmath}(0)+\int_{0}^{1} \Phi(1, t) f_{z z}\left[z_{1}, z_{\jmath}\right] d t \\
& =\int_{0}^{1} \Phi(1, t) f_{z z}\left[z_{l}(t), z_{\jmath}(t)\right] d t
\end{aligned}
$$

Using the above estimate on $\Phi(t, \tau)$ together with the observation that the components of $f_{z z}$ are etther 0 or $-V^{\prime \prime \prime}(x(t))$, we can apply Corollary 4 to conclude that $z_{i j}(t)=O\left(\left|y_{0}\right|^{-1}\right)$ for $0 \leq t \leq 1$ 
The estımate $z_{i j k}(1)=O\left(\left|y_{0}\right|^{-1}\right)$ is obtained in precisely the same way, by using all previous estımates on $z, z_{\imath}, z_{l j}$ together with Corollary 4 The last estımate $z_{i j k l}(1)=O\left(\left|y_{0}\right|^{-1}\right)$ is obtained analogously This argument makes it clear that the remainder $r$ is $C^{k}$-small for large $y_{0}$ if $V \in C^{k+1}$

\section{REFERENCES}

[1] S Aubry \& P Y LeDaeron The discrete Frenkel-Kontorova model and its extensions I Exact results for the ground states Physica 8D (1983), 381-422

[2] G D Birkhoff Dynamical Systems, Amer Math Soc Colloq Publ IX (1966), 165-169

[3] R Dieckerhoff \& E Zehnder An 'a pnori' estımate for osctllatory equation $D v n$ Sys and Bifurcations, Groningen, 1984 LNM, 1125 Springer Berlın-New York, 1985, pp 9-14

[4] R Dieckerhoff \& E Zehnder Boundedness of solution via the twist-theorem Preprint No 22/1984 Ruhr-Universitat Bochum

[5] J Franks Generalization of the Poincaré-Bırkhoff Theorem Ann Math To appear

[6] G Gruner \& A Zettl CDW conduction a novel collective transport phenomenon in solids Phys Rep 119 No 3 (March 1985), 119-232

[7] P Hartman On boundary value problems for superlinear second order differential equations $J$ Diff Eq 26 (1977), 37-53

[8] M R Herman Sur les courbes invariantes par des diffeomorphısmes de l'anneau Asterisque 1 (1983), 103-104, Asterisque 2 (1986), 144

[9] H Jacobowitz \& R A Struble Periodic solutions of $x^{\prime \prime}+f(x, t)=0$ via the Poincaré-Birkhoff theorem $J$ Diff Eq 20 No 1 (1976), 37-52 Corrigendum The existence of the second fixed point a correction to 'Penodic solutions 'above $J$ Diff Eq 25 No 1 (1977), 148-149

[10] A Katok Some remarks on the Birkhoff and Mather twist theorems Ergod Th Dynam Sys 2 (1982), 183-194

[11] G R Morris A case of boundedness in Littlewood's problem on oscillatory differential equation Bull Austr Math Soc 14 (1976), 71-93

[12] J N Mather Exıstence of quasi-penodic orbits for twist homeomorphisms of the annulus Topologv 21 (1982), 457-476

[13] J K Moser On invariant curves of area-preserving mappings of annulus Nachr Acad Wiss Gottingen Math-Phvs KI II (1962), 1-20

[14] J K Moser Break-down of stability Lect Notes in Phys 247, J M Jowett, M Month and S Turner, eds, Sprınger Berlın-New York, 1986, pp 492-518

[15] J K Moser Monotone twist mappings and the calculus of variations Ergod Th Dynam Svs 6 (1986) 401-413

[16] H Russmann Uber invarıante kurven differenzierbarer abbıldungen eines kreısringes Nachr Akad Wiss, Gottingen II, Math Phys Kl (1970) 67-105

[17] J K Moser Quasi-penodic solutions of nonlinear ellıptıc partıal differentıal equations Bol Soc Bras Mat 20(1) (1989), 29-45 